\title{
Problematic presentation and use of efficacy measures in current trials of CGRP monoclonal antibodies for episodic migraine prevention: A mini-review
}

Cephalalgia

2020, Vol. 40(I) 122-126

(C) International Headache Society 2019

Article reuse guidelines:

sagepub.com/journals-permissions DOI: $10.1177 / 0333102419877663$ journals.sagepub.com/home/cep

@SAGE

\author{
Peer Tfelt-Hansen', Hans-Christoph Diener ${ }^{2}$ and \\ Timothy J Steiner ${ }^{3,4}$
}

\begin{abstract}
Background: In trials of monoclonal antibodies against calcitonin gene-related peptide or its receptor for prevention of episodic migraine, we observed two problematic aspects: a) The graphic presentations; b) the methods of calculating "response rates" ( $\geq 50 \%$ decrease of monthly migraine days from baseline).

Observations: Decrease in monthly migraine days is presented, over time, in figures on a downward (negative) scale from zero at baseline, with the ordinate stopped just beyond the maximum effect of the active drugs. In one trial, decreases in monthly migraine days were -1.8 after placebo, -3.2 after erenumab $70 \mathrm{mg}$ and -3.7 after erenumab $140 \mathrm{mg}$, with the ordinate stopped at -4.5 . The reader can perceive only a relative 2 -fold benefit of erenumab versus placebo. If, however, treatment periods are compared with baseline in bar charts, MMDs persisting after treatment in the same trial can be illustrated as follows, creating a different perception: $78 \%$ for placebo, $61 \%$ for erenumab $70 \mathrm{mg}$, and $55 \%$ for erenumab $140 \mathrm{mg}$.

In the nine trials, "response rates" defined as above were calculated in five different ways, taking different numbers of treatment months into account in comparisons with the one-month baseline. This makes comparisons impossible.

Suggestions for improvements: Mean monthly migraine days before and after treatment should be presented in a bar chart. Such figures, presenting persisting MMDs, are more clinically relevant and less misleading than decreases from baseline.

The definition and methods of calculating and presenting " $50 \%$ response rates" should be standardized by the Drug Trial Committee of the International Headache Society.
\end{abstract}

\section{Keywords}

CGRP antibodies, episodic migraine, persisting migraine days, 50\% response rate

Date received: 13 April 2019; revised: I July 2019; accepted: 27 July 2019

\section{Introduction}

After a long period with little progress in the drugprevention of migraine, the recent development of monoclonal antibodies against CGRP or its receptor, based on improved understanding of the pathophysiology of migraine, offers a new treatment option $(1,2)$. Assuming at least equal efficacy, a likely advantage of these specific treatments is better tolerability than is experienced with the range of drugs currently used for migraine prevention. For example, in randomized, controlled trials (RCTs) of topiramate $100 \mathrm{mg}, 24 \%$ $(90 / 380)$ of patients discontinued active treatment
'Danish Headache Center, Department of Neurology, Rigshospitalet, Faculty of Health and Medical Sciences, University of Copenhagen, Glostrup, Denmark

${ }^{2}$ Medical Faculty of the University Duisburg-Essen, Essen, Germany

${ }^{3}$ Department of Neuromedicine and Movement Science, Norwegian University of Science and Technology, Trondheim, Norway

${ }^{4}$ Division of Brain Sciences, Imperial College London, London, UK

\section{Corresponding author:}

Peer Tfelt-Hansen, Danish Headache Center, Glostrup Hospital, DK2600, Glostrup, Denmark.

Email: d036218@dadlnet.dk 
because of adverse events (AEs), while 10\% (40/381) discontinued placebo (absolute difference 13\% [95\% CI: $8-18 \%$ ) (3-5). In contrast, in five large RCTs (6-10) of antibodies against CGRP or its receptor, discontinuations due to AEs were $2 \%$ both for active treatments (58/2376) and for placebo (34/1764).

However, in the various presentations and evaluations of efficacy data in the recent antibody RCTs, two problems emerge. These are reviewed in the next paragraphs.

\section{Review and comment}

The first problem, well displayed in an RCT of erenumab $70(10)$, is in the presentation of decrease in monthly migraine days (MMDs) over time on a downward (negative) scale from zero at baseline. In Figure 1 from the publication in Cephalalgia 2018 (6)), the visual impression is of migraine largely disappearing after 3 months of active treatment, as the curves flatten at MMD -2.7 to -2.9 while the ordinate is stopped at -3.5 (Figure 1). With the placebo curve levelling at MMD -1.8 , and no anchors at either end of the scale, the reader can only perceive a considerable relative benefit. The reality is as shown in Table 1: Decreases in MMDs are from 8.1 (mean baseline) to 5.2 with erenumab $70 \mathrm{mg}$ (i.e. with $64 \%$ of MMDs still persisting), and from 8.4 to 6.6 (79\% persisting) with placebo.

Patients contemplating drugs for migraine prevention need information that is both reliable and realistic. The visual presentation may appear convincing (and, presumably, is intended to be), but it is a manipulation brought about by ordinate truncation. Numerical presentation of MMDs persisting after treatment better informs both clinicians and patients. In 12 treatments with CGRP antibodies in seven phase-3 RCTs, the median persisting MMDs were 55\% (range: 45-64\%), whereas on placebo $(\mathrm{n}=7)$ the median persisting MMDs were $75 \%(69-78 \%)$ (Table 1). In summary, CGRP antibodies reduce MMDs by about a half, and placebo by about one quarter, which is a relative twofold benefit, as suggested by the visual presentation, but, importantly, a much smaller absolute benefit. An alternative visual presentation of the data in Figure 1 (10), simpler, realistic and therefore more informative, is presented in Figure 2.

The second problem lies in the different ways of calculating "response rates" in MMDs: $\geq 50 \%$ and $\geq 75 \%$; and, in some cases, in the directly erroneous way of calculating (or reporting) 100\% response rate (11). The proportion of patients with MMD reductions of $\geq 50 \%$ (the response rate) has been variously estimated/ calculated:

- as $\geq 50 \%$ mean decrease in MMDs in month 3 of 3 months' treatment after baseline (in five RCTs $(9,12,13,15,16))$

- as $\geq 50 \%$ decrease in MMDs calculated as the mean of monthly estimates during months 4-6 of 6 months' treatment after baseline (one RCT (8))

- as $\geq 50 \%$ decrease in MMDs calculated as the mean of monthly estimates during all 3 of 3 months' treatment after baseline (one RCT (10))

- as $\geq 50 \%$ mean decrease in MMDs during weeks 5-8 of 3 months' follow-up after a single intravenous injection (one RCT (14))

- as $\geq 50 \%$ decrease in MMDs calculated as the mean of monthly estimates during all 6 of 6 months' treatment after baseline (two RCTs $(6,7)$ ).

Despite these different methodologies, the $\geq 50 \%$ response rate for placebo ranged relatively narrowly

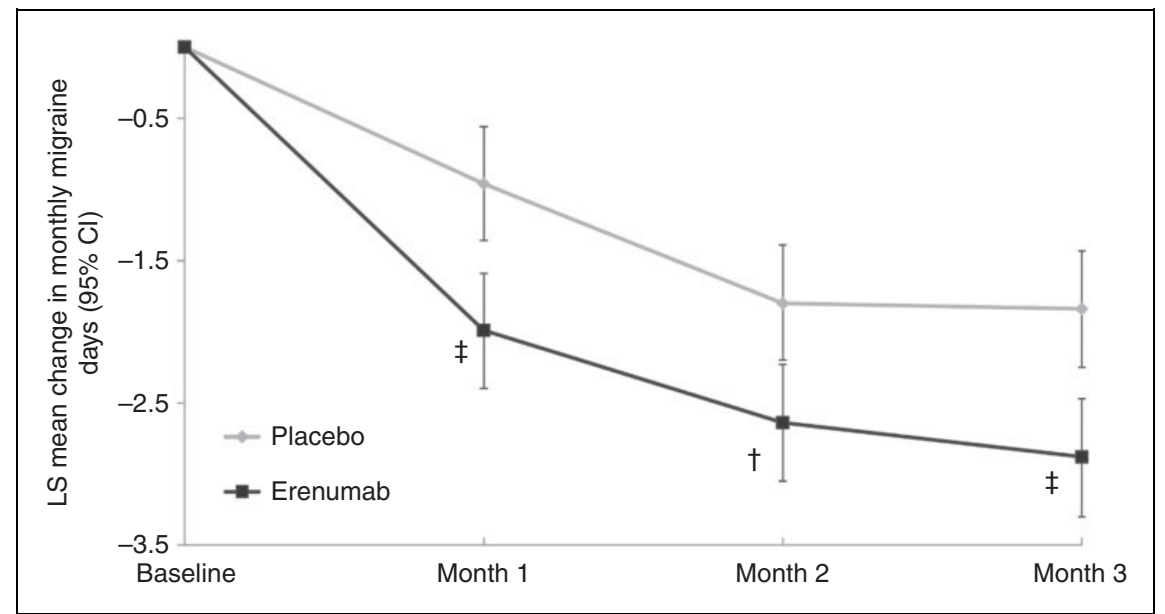

Figure I. Changes in mean monthly migraine days after treatment with erenumab $70 \mathrm{mg}$ and placebo (monthly subcutaneous injection) (9). Reproduced from Dodick et al., Cephalalgia 2018; 38: 1026-1037, with permission from the publisher. 
Table I. Preventative effects of CGRP antibodies in episodic migraine RCTs.

\begin{tabular}{|c|c|c|c|c|}
\hline Drug and dose (ref.) & $\begin{array}{l}\text { Mean MMD } \\
\text { at baseline }\end{array}$ & $\begin{array}{l}\text { Mean decrease } \\
\text { in MMD during } \\
\text { treatment }\end{array}$ & $\begin{array}{l}\text { Mean persisting } \\
\text { MMD during } \\
\text { treatment } \\
\text { (\% of baseline) }\end{array}$ & $\begin{array}{l}\text { Proportion with } \geq 50 \% \text { decrease } \\
\text { in MMD ( } 50 \% \text { response rate) }\end{array}$ \\
\hline Erenumab $70 \mathrm{mg}(\mathrm{n}=282)(9)$ & 8.1 & -2.9 & $5.2(64 \%)$ & $40 \% ; \mathrm{TG}=10 \%(95 \% \mathrm{Cl}: 2-18 \%)$ \\
\hline Placebo $(n=288)$ & 8.4 & -1.8 & $6.6(79 \%)$ & $30 \%$ \\
\hline Erenumab $70 \mathrm{mg}(\mathrm{n}=3 \mid 8)(8)$ & 8.3 & -3.2 & $5.1(61 \%)$ & $43 \% ; \mathrm{TG}=17 \%$ (95\% Cl: 9-24\%) \\
\hline Erenumab $140 \mathrm{mg}(\mathrm{n}=318)$ & 8.3 & -3.7 & $4.6(55 \%)$ & $50 \% ; \mathrm{TG}=23 \%(95 \% \mathrm{Cl}: 16-31 \%)$ \\
\hline Placebo $(n=319)$ & 8.2 & -1.8 & $6.4(78 \%)$ & $27 \%$ \\
\hline Erenumab $70 \mathrm{mg}(\mathrm{n}=104)(12)$ & 8.6 & -3.4 & $5.2(60 \%)$ & $46 \% ; \mathrm{TG}=17 \%$ (95\% Cl: 4-29\%) \\
\hline Placebo $(n=153)$ & 8.8 & -2.3 & $6.5(74 \%)$ & $30 \%$ \\
\hline Fremazenumab $225 \mathrm{mg}(\mathrm{n}=289)(10)$ & 9.1 & -3.7 & $5.4(59 \%)$ & $48 \% ; \mathrm{TG}=20 \%(95 \% \mathrm{Cl}: \quad 12-27 \%)$ \\
\hline Fremazenumab $675 \mathrm{mg}(\mathrm{n}=289)$ & 9.3 & -4.4 & $4.9(53 \%)$ & $44 \% ; \mathrm{TG}=16 \%(95 \% \mathrm{Cl}: 9-24 \%)$ \\
\hline Placebo $(n=294)$ & 9.1 & -2.2 & $6.9(76 \%)$ & $28 \%$ \\
\hline Fremazenumab $225 \mathrm{mg} / \mathrm{mo}(\mathrm{n}=96)(13)$ & 11.5 & -6.3 & $5.2(45 \%)$ & $53 \% ; \mathrm{TG}=25 \%(95 \% \mathrm{Cl}: \mathrm{II}-38 \%)$ \\
\hline Fremazenumab $225 \mathrm{mg}$ once $(\mathrm{n}=97)$ & 11.3 & -6.1 & $5.2(46 \%)$ & $59 \% ; \mathrm{TG}=32 \%(95 \% \mathrm{Cl}: 14-44 \%)$ \\
\hline Placebo $(n=104)$ & 11.5 & -3.5 & $8.0(70 \%)$ & $28 \%$ \\
\hline Eptinezumab $1000 \mathrm{mg}$ (i.v.) $(\mathrm{n}=8 \mathrm{I})(\mathrm{I} 4)$ & 8.1 & -5.6 & $2.5(31 \%)$ & $75 \% ; \mathrm{TG}=22 \%$ (95\% Cl: 7-35\%) \\
\hline Placebo $(n=82)$ & 8.2 & -4.6 & $3.6(44 \%)$ & $54 \%$ \\
\hline Galcanezumab $120 \mathrm{mg}(\mathrm{n}=210)(6)$ & 9.2 & -4.7 & 4.5 (49\%) & $62 \% ; \mathrm{TG}=24 \%(95 \% \mathrm{Cl}: \quad 15-32 \%)$ \\
\hline Galcanezumab $240 \mathrm{mg}(\mathrm{n}=210)$ & 9.1 & -4.6 & $4.5(49 \%)$ & $61 \% ; \mathrm{TG}=22 \%(95 \% \mathrm{Cl}: 14-30 \%)$ \\
\hline Placebo $(n=425)$ & 9.1 & -2.8 & $6.3(69 \%)$ & $39 \%$ \\
\hline Galcanezumab $120 \mathrm{mg}(\mathrm{n}=23 \mathrm{I})(7)^{\mathrm{a}}$ & 9.1 & -4.3 & $4.8(53 \%)$ & $59 \% ; \mathrm{TG}=23 \%(95 \% \mathrm{Cl}: \quad 15-31 \%)$ \\
\hline Galcanezumab $240 \mathrm{mg}(\mathrm{n}=223)$ & 9.1 & -4.2 & $4.9(54 \%)$ & $57 \% ; \mathrm{TG}=20 \%$ (95\% Cl: I3-28\%) \\
\hline Placebo $(n=46 \mathrm{I})$ & 9.2 & -2.3 & 6.9 (75\%) & $36 \%$ \\
\hline Galcanezumab $120 \mathrm{mg}(\mathrm{n}=62)(15)$ & 6.7 & -4.8 & $1.9(28 \%)$ & $\begin{array}{l}76 \% ; \mathrm{TG}=14 \% \\
\quad(95 \% \mathrm{Cl}:-0.5-26 \%)\end{array}$ \\
\hline Placebo $(n=126)$ & 6.6 & -3.7 & $2.9(44 \%)$ & $62 \%$ \\
\hline Erenumab 140 mg $(\mathrm{n}=119)(16)$ & 9.2 & -1.8 & $7.4(80 \%)$ & $30 \% ; \mathrm{TG}=17 \%(95 \% \mathrm{Cl}: 6-27 \%)$ \\
\hline Placebo $(n=124)$ & 9.3 & -0.2 & 9.1 (98\%) & $14 \%$ \\
\hline
\end{tabular}

${ }^{a}$ Secondary outcome measures in one large RCT with galcanezumab (7): "The mean proportion of patients with reduction from baseline of 50\%, 75\%, and $100 \%$ in monthly MHDs during the 6-month double-blind treatment phase. Specifically, response rate was defined as the percentages of patients meeting predefined thresholds (i.e. $50 \%, 75 \%$, and 100\%) in the reduction from baseline in the number of MHDs for each month, and the overall percentages of patients meeting these thresholds averaged over months I through 6 were analyzed".

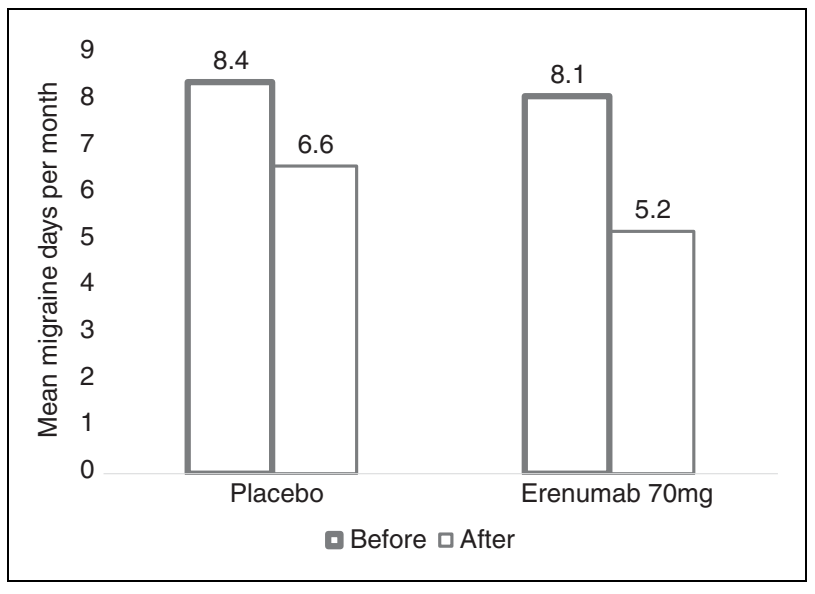

Figure 2. Mean migraine days per month before and after treatment with placebo or erenumab $70 \mathrm{mg}$ (monthly subcutaneous injection) for 3 months (9).
$(27-39 \%)$ in the seven phase-3 RCTs, with a median of $30 \%(6-10,15,16)$. It is perhaps noteworthy that the two greatest placebo MMD reductions, $36 \%$ and $39 \%$, were observed in the two galcanezumab RCTs $(6,7)$, with the very special methodology of calculating response in each month over all 6 months of treatment (see notes to Table 1). In the two smaller phase-2 RCTs, the $\geq 50 \%$ placebo response rates were, for unknown reasons, $54 \%$ (14) and $62 \%$ (15).

The only recent, previous trial programme of a preventative drug for episodic migraine was conducted with topiramate $(n=1367)$, with similar methodology in three RCTs (3-5). The optimum dose of topiramate was $100 \mathrm{mg}$, which was tested in 378 patients who were compared with 378 treated with placebo. A 4-week baseline period was followed by an 8-week titration period and an 18-week maintenance period, with 
$\geq 50 \%$ decrease in MMDs from baseline evaluated over the whole treatment period. Response rates were $47.5 \%$ on topiramate and $22.5 \%$ on placebo; the therapeutic gain (TG) (active drug minus placebo) was $25 \%(95 \%$ CI: $18-32 \%)$. Thus, the best results from treatment with CGRP antibodies match the TG for topiramate, an established drug for migraine prevention, but the diverse calculations of $\geq 50 \%$ response rates in the CGRP RCTs exclude fair comparison. There is therefore a need for comparative RCTs between the CGRPrelated antibodies and standard drug treatments.

Estimates and reporting of $75 \%$ response rate, defined as the proportion of treated patients with $\geq 75 \%$ decrease in MMDs $(6,7,13,16)$, probably suffer from the same problems, while $100 \%$ response rate, defined as the proportion of treated patients with a $100 \%$ decrease of MMD (i.e. migraine-free), has been reported in a peculiar way in two studies $(6,7)$ (see below).

In one large phase-3 galcanezumab RCT (6), 100\% response rates were $6.2 \%(26 / 425)$ for placebo, $15.6 \%$ $(33 / 210)$ for galcanezumab $120 \mathrm{mg}$ (odds ratio [OR]: 2.8 [95\% CI: $2.0-4.0]$ ) and $14.6 \%$ (30/210) for galcanezumab $240 \mathrm{mg}$ (OR: 2.6 [95\% CI: 1.8-3.7]). In the other phase-3 galcanezumab RCT (7), 100\% response rates were similar: $26 / 461(5.7 \%)$ for placebo, $27 / 231$ $(11.5 \%)$ for galcanezumab $120 \mathrm{mg}$, and $31 / 223$ $(13.8 \%)$ for galcanezumab $240 \mathrm{mg}$ (for how these secondary outcome parameters were evaluated, see note in Table 1). Apparently, there were consistent findings of a small but possibly relevant effect of the two galcanezumab doses. In a post hoc analysis of $100 \%$ response rates on galcanezumab $120 \mathrm{mg}$ and $240 \mathrm{mg}$ and placebo in these two phase-3 RCTS (17), the results were presented in an alternative way: As the number of months with $100 \%$ response (17). At least 1 month migrainefree during 6 months' treatment was observed in 171/ $875(19.5 \%)$ of patients on placebo, $169 / 436(38.8 \%)$ on galcanezumab $120 \mathrm{mg}$ and $178 / 428(41.6 \%)$ on galcanezumab $240 \mathrm{mg}$. Three months migraine-free were observed in $48 / 875(5.5 \%)$ on placebo, 46/436 $(10.6 \%)$ on galcanezumab $120 \mathrm{mg}$ and $59 / 428(13.8 \%)$ on galcanezumab $240 \mathrm{mg}$. Finally, $100 \%$ response during all 6 months was observed in $2 / 827(0.2 \%)$ on placebo, $3 / 435(0.7 \%)$ on galcanezumab $120 \mathrm{mg}$ and $6 /$ $427(1.4 \%)$ on galcanezumab $240 \mathrm{mg}$ (17). In our view,
" $100 \%$ response" describes freedom from migraine attacks for a predefined period within or after the treatment period, not random months of migraine freedom dispersed among six months (11). The galcanezumab RCTs demonstrate that $100 \%$ response over all 6 months occurs very seldom (in approximately $1 \%$ of patients).

\section{Conclusions}

Changes in MMDs in RCTs of migraine preventative drugs should be presented graphically, in an appropriate way that provides realistic information to patients and their doctors, not manipulated to emphasize differences from placebo. This is best done by presenting baseline MMDs and MMDs persisting after a period of treatment.

The $50 \%$ response rate (proportion with $\geq 50 \%$ decrease in MMDs) is an important secondary outcome measure, recommended by the International Headache Society (IHS) since 2012 (18). When no RCTs directly comparing two drugs for migraine prevention are available, their relative effects can, with some caution, be estimated by comparing their TGs on this measure. This outcome has also been used in meta-analyses of propranolol (19) and topiramate (20). Generally, a prerequisite for using an outcome measure for comparisons is, of course, that it is similarly calculated in all RCTs, and this is not the case for CGRP antibody RCTs: Investigators and manufacturers of these drugs have used five different ways to calculate the $50 \%$ response rate. The reason for this is obscure, but the consequence is that it is very doubtful whether this measure can be used to compare the CGRP antibodies with each other or with other preventative drugs. Given the enormous investment in these RCTs, this is regrettable (and, arguably, unforgivable). An additional caveat is that in most earlier RCTs, with other agents, 50\% response rates were estimated as $\geq 50 \%$ decrease in migraine attacks per month (regardless of attack duration), not migraine days (MMDs: All days affected by or during the course of attacks) as used in the CGRP antibody RCTs.

We hope that the IHS Drug Trial Committee will formulate new and more precise recommendations concerning the definition and reporting of response rates in RCTs investigating the prevention of (episodic) migraine.

\section{Clinical implications}

- Current illustrations of effect of CGRP antibodies in migraine can give an exaggerated impression of the effect.

- This is due to the presentation only of changes in monthly migraine days (MMDs) for placebo and active drugs.

- Presentation of mean MMDs during baseline and persisting MMDs after treatment will provide more relevant information.

- In the trials with CGRP antibodies, $\geq 50 \%$ decrease in MMDs is calculated in five different ways, and a consensus on how this should be done is needed. 


\section{Declaration of conflicting interests}

The authors declared the following potential conflicts of interest with respect to the research, authorship, and/or publication of this article: $\mathrm{H}-\mathrm{CD}$ received honoraria for participation in clinical trials, contribution to advisory boards or oral presentations from: Alder, Allergan, Amgen, Autonomic Technology, Bristol-Myers Squibb, CoLucid, Electrocore, Ipsen, Lilly, Medtronic, MSD, Novartis, Pfizer, Schaper and Brümmer, Teva and Weber \& Weber. Financial support for research projects was provided by Allergan, Electrocore, MSD and Pfizer. Headache research at the Department of Neurology in Essen is supported by the German Research Council (DFG), the German Ministry of Education and Research (BMBF) and the European Union. H-CD has no ownership interest and does not own stocks of any pharmaceutical company. H-CD serves on the editorial boards of Cephalalgia and Lancet Neurology. H-C Diener chairs the Clinical Guidelines Committee of the German Society of Neurology and is member of the Clinical Trials Committee of the IHS. PT-H and TS have no conflicts of interests.

\section{Funding}

The authors received no financial support for the research, authorship, and/or publication of this article.

\section{References}

1. Edvinsson L, Haanes KA, Warfvinge K, et al. CGRP as the target of new migraine therapies - successful translation from bench to clinic. Nat Rev Neurol 2018; 14: 338-350.

2. Ong JJY, Wei DY and Goadsby PJ. Recent advances in pharmacotherapy for migraine prevention: From pathophysiology to new drug. Drugs 2018; 8: 411-437.

3. Brandes JL, Saper JR, Diamond M, et al. Topiramate for migraine prevention: A randomized controlled trial. JAMA 2004; 291: 965-973.

4. Silberstein SD, Neto W, Schmitt J, et al.; MIGR-001 Study Group. Topiramate in migraine prevention: Results of a large controlled trial. Arch Neurol 2004; 61: 490-495.

5. Diener H, Tfelt-Hansen P, Dahlöf C, et al. Topiramate in migraine prophylaxis - results from a placebo-controlled trial with propranolol as an active control. J Neurol 2004; 251: 943-950.

6. Stauffer VL, Dodick DW, Zhang Q, et al. Evaluation of galcanezumab for the prevention of episodic migraine: The EVOLVE-1 randomized clinical trial. JAMA Neurol 2018; 75: $1080-1088$.

7. Skljarevski V, Matharu M, Millen BA, et al. Efficacy and safety of galcanezumab for the prevention of episodic migraine: Results of the EVOLVE-2 Phase 3 randomized controlled clinical trial. Cephalalgia 2018; 38: $1442-1454$.
8. Goadsby PJ, Reuter U, Hallstrom Y, et al. A controlled trial of erenumab for episodic migraine. $N$ Engl J Med 2017; 377: 2123-2132.

9. Dodick DW, Ashina M, Brandes JL, et al. ARISE: A Phase 3 randomized trial of erenumab for episodic migraine. Cephalalgia 2018; 38: 1026-1037.

10. Dodick DW, Silberstein SD, Bigal ME, et al. Effect of fremanezumab compared with placebo for prevention of episodic migraine: A randomized clinical trial. JAMA 2018; 319: 1999-2008.

11. Steiner TJ and Tfelt-Hansen P. Misused and misleading " $100 \%$ response rate" to galcanezumab in patients with episodic migraine. Headache 2019; 59: 626.

12. Sun H, Dodick DW, Silberstein S, et al. Safety and efficacy of AMG 334 for prevention of episodic migraine: A randomised, double-blind, placebo-controlled, phase 2 trial. Lancet Neurol 2016; 15: 382-390.

13. Bigal ME, Dodick DW, Rapoport AM, et al. Safety, tolerability, and efficacy of TEV-48125 for preventive treatment of high-frequency episodic migraine: A multicentre, randomised, double-blind, placebo-controlled, phase $2 \mathrm{~b}$ study. Lancet Neurol 2015; 14: 1081-1090.

14. Dodick DW, Goadsby PJ, Silberstein SD, et al. Safety and efficacy of ALD403, an antibody to calcitonin generelated peptide, for the prevention of frequent episodic migraine: A randomised, double-blind, placebo-controlled, exploratory phase 2 trial. Lancet Neurol 2014; 13: $1100-1107$.

15. Skljarevski V, Oakes TM, Zhang Q, et al. Effect of different doses of galcanezumab vs placebo for episodic migraine prevention: A randomized clinical trial. JAMA Neurol 2017; 75: 187-193.

16. Reuter U, Goadsby PJ, Lanteri-Minet M, et al. Efficacy and tolerability of erenumab in patients with episodic migraine in whom two-to-four previous preventive treatments were unsuccessful: A randomised, double-blind, placebo-controlled, phase 3b study. Lancet 2018; 392: 2280-2287.

17. Rosen N, Pearlman E, Ruff D, et al. $100 \%$ response rate to galcanezumab in patients with episodic migraine: A post hoc analysis of the results from Phase 3, randomized, double-blind, placebo-controlled EVOLVE-1 and EVOLVE-2 studies. Headache 2018; 58: 1347-1357.

18. Tfelt-Hansen P, Pascual J, Ramadan N, et al. International Headache Society Clinical Trials Subcommittee. Guidelines for controlled trials of drugs in migraine: Third edition. A guide for investigators. Cephalalgia 2012; 32: 6-38.

19. Linde $\mathrm{K}$ and Rossnagel K. Propranolol for migraine prophylaxis. Cochrane Database Syst Rev 2004; 2: CD003225.

20. Linde M, Mulleners WM, Chronicle EP, et al. Topiramate for the prophylaxis of episodic migraine in the adults. Cochrane Database Syst Rev 2013; 6: CD010610. 
This text is made available via DuEPublico, the institutional repository of the University of Duisburg-Essen. This version may eventually differ from another version distributed by a commercial publisher.

DOI: $\quad 10.1177 / 0333102419877663$

URN: urn:nbn:de:hbz:464-20210510-123335-2

This publication is with permission of the rights owner freely accessible due to an Alliance licence and a national licence (funded by the DFG, German Research Foundation) respectively.

(C) International Headache Society 2019. All rights reserved. 